J. Lake Sci. (湖泊科学) , 2018, 30(3): 753-762

DOI 10. 18307/2018. 0317

(c) 2018 by Journal of Lake Sciences

\title{
产卵迁徙对金沙江黑水河下游鱼类群聚结构变动的影响”
}

\author{
杨 志, 唐会元 * , 龚 云, 朱 迪, 赵 娜 \\ (水利部中国科学院水工程生态研究所,水利部水工程生态效应与生态修复重点实验室,武汉 430079)
}

\begin{abstract}
摘 要: 鱼类作为河流生物的重要组成部分, 其在干支流之间的迁徙或移动不仅是常见的,而且通常与鱼类的繁殖活动 相联系. 金沙江下游支流作为金沙江下游河流网络的重要组成部分, 分布有较为丰富的集合生境以及较高的鱼类物种多 样性. 研究鱼类在金沙江下游干支流的产卵迁徙对支流鱼类群聚结构变动的影响以及支流生境维持对区域鱼类种群维 系的意义,对金沙江下游干支流鱼类的保护具有重要的意义. 本文拟选择黑水河下游江段作为典型研究区域,通过 2014 年在该区域的逐月渔获物调查,采用聚类分析、基于距离的线性模型以及基于距离的圥余分析等多种多元分析方法, 确 定黑水河下游群聚结构的逐月变动是否严重依赖于鱼类在干支流之间的产卵迁徙以及黑水河下游自然生境的维持对区 域鱼类种群的维系是否具有重要的意义. 结果表明:黑水河下游鱼类群聚结构在金沙江雨季和旱季显著分离的同时,表 征这种分离的 8 种指示种的性成熟个体丰度也在雨季和旱季间发生不同程度的变动. 7 种指示种鱼类性成熟个体丰度的 变动能够解释黑水河下游鱼类群聚结构变动 $77.20 \%$ 的变异, 其中齐口裂腹鱼、大鳞副泥鱾和犁头䲝性成熟个体丰度的变 动是影响黑水河下游鱼类群聚结构变动的 3 个最显著的因素, 7 种指示种鱼类在干支流的产卵迁徙对黑水河下游鱼类群 聚结构的变动造成了明显的影响. 研究表明: 在金沙江干支流严重水电开发背景下, 维持黑水河现有的自然生境面积对 区域鱼类种群的维持具有重要意义. 为实行上述目标, 建议在白鹤滩水电站蓄水运行后, 拆除黑水河的部分小型水坝, 并 采取其他河流再自然化措施以维持黑水河现有的自然生境面积.
\end{abstract}

关键词: 金沙江下游;黑水河;栖息地保护;河流连通性;指示种;基于距离的线性模型

\section{Effect of spawning migration on the variations of fish assemblage structures in the lower reaches of the Heishui River, Jinsha River}

YANG Zhi, TANG Huiyuan ${ }^{* *}$, GONG Yun, ZHU Di \& ZHAO Na

(Key Laboratory of Ecological Impacts of Hydraulic-projects and Restoration of Aquatic Ecosystem of Ministry of Water Resources, Institute of Hydroecology, Ministry of Water Resources and Chinese Academy of Sciences, Wuhan 430079, P.R.China)

Abstract: Fishes are the important parts of aquatic ecosystems in the rivers. Fish migration or movement between mainstream and tributaries is not only common, but also usually closely associated with fish spawning activity. As an important component of the Jinsha River network, the lower reaches of the Jinsha River exhibit relatively abundance meta-habitats and higher fish species diversity. Studying the effect of fish spawning migration between the mainstream of the Jinsha River and its tributaries on the variations of fish assemblages in the tributaries, as well as the importance of tributary habitat maintenance for the preserve of local fish population size, are of great significances to protect the fish resources of the tributaries of the Jinsha River. Heishui River is one of the important tributaries in the lower reaches of the Jinsha River. In this study, we selected some river segments of Heishui River as a typical study region. Based on the monthly fish catch investigations in this study region in 2014 , a variety of multivariate analysis methods including cluster analysis, distance-based linear model, distance-based redundancy analysis and other statistic methods were used for: determining whether the monthly changes in fish assemblage structures of the lower reaches of the Heishui River were heavily dependent on fish spawning migration between the mainstream of the Jinsha River and the lower reaches of the Heishui River, and determining whether the natural habitat of the lower reaches in the Heishui River was important for the maintenance of

* 国家自然科学基金项目(51379134)、国家重点研发计划项目(2016YFC0502206) 和水利部公益性行业科研专项经 费项目 (201501001) 联合资助. 2017-06-01 收稿; 2017-08-14 收修改稿. 杨志 (1982 ), 男, 硕士, 助理研究员; E-mail: yangzhi4626@163.com.

** 通信作者;E-mail:tanghy@163.com. 
the local fish populations. The results showed that the fish assemblage structures of the lower reaches of the Heishui River were significantly separated in the rainy and dry seasons of the Jinsha River. This separation resulted from the significantly differences in abundances of eight indicator species between the rainy and dry seasons in the Jinsha River. Correspondingly, the abundances of sexual mature individuals of these eight species also showed some changes between the rainy and dry seasons. The variations in abundances of sexual mature individuals of seven indicator species could explain $77.20 \%$ of the total variations of fish assemblage structures of the lower reaches of the Jinsha River, herein the variations in abundances of sexual mature individuals of Schizothorax prenanti , Lepturichthys fimbriata and Paramisgurnus dabryanus were the three most significant factors affecting the variations of fish assemblage structures of the lower reaches of the Heishui River. Overall, the spawning migrations of seven indicator species have the significant impacts on the variations of fish assemblage structures of the lower reaches of the Heishui River. This study showed that under the background of serious hydropower developments in the mainstem and tributaries of the Jinsha River, keeping the existing natural habitat areas of the lower reaches of the Heishui River is very important for maintaining fish population survival of the lower reaches of the Jinsha River. For the implementation of the above objective, after the normal operation of the Baihetan reservoir, the removals of some small dams as well as other river re-naturalizing measures should be applied for maintaining the existing natural habitat area of the Heishui River.

Keywords: The lower reaches of the Jinsha River; Heishui River; habitat protection; rive connectivity; indicator species; distance-based linear model

河流作为一个由干流和各级支流共同组成的复杂网络结构 ${ }^{[1-2]}$, 其内部发生的输运过程在河流流域水 文、生物和生态过程的相互作用中扮演了重要的角色 ${ }^{[3]}$. 在此过程中, 等级化以及分支化的河流网络与动态 的流域扰动相作用,导致河流生境在时空上的不均匀分布,并最终决定了不同区域的生物多样性和生产 力 ${ }^{[2,4]}$. 而鱼类作为河流生物及其生产力的重要组成部分, 往往具有在不同栖息生境之间进行主动迁徙或移 动的能力 ${ }^{[5-6]}$. 特别地, 对许多河流而言, 其干支流之间鱼类的迁徙或移动不仅是常见的, 而且通常与鱼类的 繁殖活动相联系 ${ }^{[7-9]}$. 在此情况下, 支流鱼类的群聚结构很可能会随着鱼类在干支流的迁徙或移动而显著变 动 ${ }^{[9-10]}$. 研究鱼类产卵迁徙对某一支流鱼类群聚结构变动的影响, 不仅有助于加深对这条支流群聚结构变动 模式的理解 ${ }^{[11-12]}$, 而且能够明确保持这一支流与干流联通性对区域鱼类种群维持的重要性, 有利于优先保 护支流的选择 ${ }^{[13-14]}$. 而且, 在辨识鱼类产卵迁徙对支流鱼类群聚结构影响的同时, 通过对产卵鱼类种类和数 量的分析,也可以确定支流保护对区域鱼类资源维持的意义,并能够为有针对性地提出保护措施 (例如河流 连通性恢复、设立保护区等) 保护某些重要鱼类提供基础数据支撑 ${ }^{[15-16]}$.

金沙江下游作为长江上游的重要组成部分, 具有众多的支流、较为丰富的集合生境以及较高的鱼类物 种多样性 ${ }^{[13]}$. 其中较大的一级支流有 15 条, 共分布有长江上游特有鱼类 23 种, 占金沙江下游特有鱼类总种 数的 $39.66 \%$, 为长江上游特有鱼类重要的分布区域 ${ }^{[13]}$. 这些支流作为金沙江下游河流网络的重要组成部 分,其自然生境的维持,特别是产卵场的维持对金沙江下游干流部分鱼类种群的维系也许具有重要的意义. 关于这方面的研究, 迄今为止, 还未有报道加以证实. 而且, 金沙江下游支流的鱼类群聚结构变动是否受鱼 类干支流产卵迁徙的影响也需要进行深人研究.

黑水河位于金沙江左岸, 全长 $173.3 \mathrm{~km}$, 流域面积 $3653 \mathrm{~km}^{2}$, 是金沙江下游 15 条主要支流中的一条, 为 白鹤滩库区干流鱼类的重要替代生境和优先保护支流 ${ }^{[13]}$. 白鹤滩蓄水前, 黑水河下游老木河电站坝址至河 口江段保持自然流水生境, 分布着众多的鱼类种类, 为上述科学问题的探索提供了良好的研究场所 ${ }^{[13,17]}$. 本 研究拟通过在 2014 年对黑水河下游的逐月渔获物进行调查, 首先获得黑水河下游鱼类种类组成和丰度以 及性成熟个体丰度的逐月数据, 然后采用聚类分析以及相似性检验 (analysis of similarity test, ANOSIM) 确定 鱼类群聚结构在不同月份上的聚类情况, 并在此聚类结果上获得不同聚类组的指示种组成,最后基于距离 的线性模型方法 (distance-based linear model, DistlLM) 分析群聚结构的逐月变动与指示种性成熟个体丰度 逐月变动之间的关系,并采用基于距离的圥余分析方法 (distance-based redundancy analysis, dbRDA) 对 DistlLM 结果进行可视化. 本文旨在确定: 1 ) 黑水河下游群聚结构的逐月变动是否严重依赖于鱼类在干支流 之间的产卵迁徙;2) 黑水河下游自然生境的维持对区域鱼类种群的维系是否具有重要意义. 


\section{1 材料与方法}

\section{1 调查区域与调查方法}

调查区域包括黑水河下游老木河电站 坝下约 $40 \mathrm{~km}$ 的干支流河段 (图 1). 黑水河 下游多年平均流量 $70.0 \mathrm{~m}^{3} / \mathrm{s}$, 其径流年内分 布不均, 其中汛期 (6-10月) 径流量占年径 流量的 $73.2 \%$, 而枯季 (12 月至翌年 5 月) 仅占年径流量的 $19.7 \%{ }^{[17]}$. 调查区域主要 为急流砾石生境 ${ }^{[17]}$, 非汛期时其断面平均 流速通常在 1.1 2.0 m/s 之间, 断面平均水 深通常在 $0.8 \sim 1.7 \mathrm{~m}$ 之间. 调查区域上游分 布有 4 个梯级水电站, 从上至下分别为苏家 湾、公德房、松新和老木河水电站, 其坝高分 别为 $5.1 、 18.0 、 5.3$ 和 $5.0 \mathrm{~m}^{[18]}$. 调查区域位 于老木河水电站下游. 调查时水温的分布范 围为 $10.1 \sim 24.4^{\circ} \mathrm{C}$, 平均水温 $18.1^{\circ} \mathrm{C}$. 根据不 同江段微生境的差异, 2014 年 1-12 月在 调查区域设置采样点 7 个, 并采用背负式电 捕鱼器 (电瓶: $20 \mathrm{~A}, 12 \mathrm{~V}$; 电鱼器: $3000 \mathrm{~W}$ ) 进行逐月采样( 图 1), 每月中旬采集渔获物 $4 \sim 5 \mathrm{~d}$, 每天采集约 $6 \mathrm{~h}$. 每个采样点采集河 段距离约 $2 \mathrm{~km}$. 对所有采集到的鱼类标本 进行种类鉴定, 并测量每尾个体的全长、体 长 (精确到 $1 \mathrm{~mm}$ ) 和体重 (精确到 $0.1 \mathrm{~g}$ ). 样

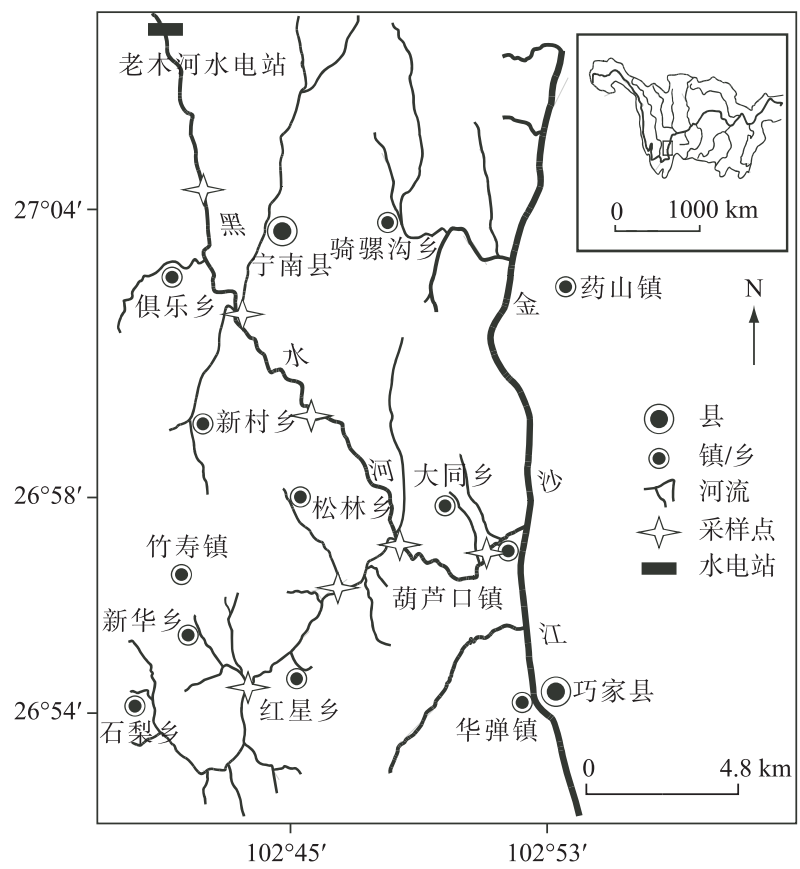

图 1 黑水河下游调查采样点分布

Fig.1 Distribution of the sampling sites in the lower reaches of the Heishui River 本采集后，随机抽样每种鱼类约 $50 \%$ $100 \%$ 个体进行解剖, 观测鱼类的性腺发育期, 并参照王剑伟 ${ }^{[19]}$ 的标准, 将 IV 期及其以上发育期个体认定为 性成熟个体. 性腺发育期的分类标准参考殷名称 ${ }^{[11]}$ 所描述的方法.

\section{2 数据分析}

1.2.1 群聚结构分析 首先计算不同鱼类种类及其性成熟个体在各月的单位捕捞努力量的渔获量 (catch per unit effort, CPUE), 其计算公式为: $C P U E_{i s}=N_{i s} / H_{i}$, 其中, $C P U E_{i s}$ 为第 $i$ 月每小时采集到第 $s$ 种鱼类的尾数或 性成熟个体尾数 (尾/h); $N_{i s}$ 为第 $i$ 月采集到第 $s$ 种鱼类的尾数或性成熟个体尾数; $H_{i}$ 为第 $i$ 月的总采样时间 ( h) , 然后基于不同鱼类种类的 CPUE, 以 Bray-Curtis 相似性系数为基础构建不同月份之间的相似性矩阵,采 用等级聚类分类方法 (cluster analysis, CA) 分析调查区域不同月份鱼类群聚结构的聚类情况 ${ }^{[20]}$. 采用 Oneway ANOSIM 方法检验各组之间群聚结构的差异是否显著 ${ }^{[20]}$.

1.2.2 指示种分析 指示种是最能代表某一类群生境特征的种类,其已在群落生态学中得到广泛的应用 ${ }^{[21]}$. 通过计算指示值 (IndVal), 各组指示种的组成得以明确. 这里的各组为群聚结构分析的结果. 指示值的计算 公式为: $I n d V a l_{i j}=100 A_{i j} \cdot B_{i j}$, 其中, $I n d V a l_{i j}$ 为物种 $i$ 在组 $j$ 中的指示值, $A_{i j}$ 为物种 $i$ 在组 $j$ 中的平均数量占其在 各个组平均数量之和的比例, 当物种 $i$ 仅在组 $j$ 中发现时, $A_{i j}$ 值最大为 $1 ; B_{i j}$ 为物种 $i$ 在组 $j$ 中出现的月份数 占组 $j$ 总月份数的比例, 当物种 $i$ 在组 $j$ 中的各月均出现时, $B_{i j}$ 值最大为 $1^{[22-23]}$. 通过软件自动迭代, 不同组 之间的月份随机重分配过程被用来检验指示种的显著性. 某一物种在某一组的指示值与其在其他组的指示 值在统计学上显著差异时,该物种才能被认定为指示种 ${ }^{[22-23]}$.

1.2.3 群聚结构的逐月变动与指示种性成熟个体丰度逐月变动的关系 基于各个月份指示种性成熟个体的 丰度 (CPUE) 以及各个月份鱼类群聚结构的丰度 (CPUE), 采用 DistlLM 构建鱼类群聚结构的逐月变动与指 
示种性成熟个体丰度逐月变动的关系模型, 其中指示种的性成熟个体丰度为自变量而鱼类群聚结构的丰度 为因变量. 采用逐步回归的方法选择模型参数, 并使用 Adjusted $R^{2}$ 评判模型的优劣 ${ }^{[24]}$. 进一步采用 dbRDA 分析对 DistlLM 结果进行可视化 ${ }^{[24]}$.

1.2.4 分析软件 采用 PRIMER 6 软件(版本 1.0.6) 进行 CA、One-way ANOSIM、DistlLM 以及 dbRDA 分析, 并 使用 CorelDRAW 12.0 和 R 软件 (版本 R.i386 3.0.1) 进行绘图. 本文的显著性水平设置为 $P<0.05$.

\section{2 结果}

\section{1 群聚结构}

2014 年 1-12 月共统计渔获物 $71.99 \mathrm{~kg}, 10531$ 尾,鉴定出种类 28 种,隶属于 3 目 8 科 23 属(表 1). 28 种鱼类中,采集尾数排在前 3 位的种类为短体副鳅、红尾副鳅和前鯺高原鳅,共采集到 9293 尾, 占采样期间 总采样尾数的 $88.24 \%$. 渔获物中, 除墨头鱼、中华倒刺鲃、齐口裂腹鱼、短须裂腹鱼、昆明裂腹鱼、鲇和瓦氏 黄颡鱼外, 其他 22 种鱼类的平均体重均小于 $50 \mathrm{~g}$. 采样期间, 某一种类性成熟个体的平均体重均明显大于 该物种所有个体的平均体重 (表 1 ).

\section{表 12014 年黑水河下游鱼类种类组成及其基础生物学参数}

Tab.1 Species composition and basic biological parameters of fishes collected from the lower reaches of Heishui River in 2014

\begin{tabular}{|c|c|c|c|c|c|c|}
\hline 种名( 拉丁名) & 尾数/尾 & 重量/g & $\begin{array}{l}\text { 平均体 } \\
\text { 重/g }\end{array}$ & $\begin{array}{l}\text { 解剖样 } \\
\text { 本数/尾 }\end{array}$ & $\begin{array}{c}\text { 性成熟个体 } \\
\text { 尾数/尾 }\end{array}$ & $\begin{array}{l}\text { 性成熟个体 } \\
\text { 平均体重 } / \mathrm{g}\end{array}$ \\
\hline 短体副鳅 (Paracobitis potanini) & 4079 & 14186.1 & 3.5 & 1876 & 672 & 7.5 \\
\hline 红尾副鲀 ( Paracobitis varigatus) & 2770 & 11416.6 & 4.1 & 1450 & 711 & 6.5 \\
\hline 泥鳅 (Misgurnus anguillicaudatus & 2 & 33.5 & 16.8 & 2 & 0 & - \\
\hline 大鳞副泥鳅 (Paramisgurnus dabryanus & 40 & 209 & 5.2 & 40 & 8 & 9.8 \\
\hline 前鳍高原鱾 (Triplophysa anterodorsalis) & 2444 & 9274.7 & 3.8 & 1579 & 763 & 6.8 \\
\hline 宽鯺鱲 (Zacco platypus) & 7 & 206.8 & 29.5 & 7 & 1 & 34.5 \\
\hline 高体近红鲌( Ancherythroculter kurematsui) & 2 & 4.1 & 2.1 & 2 & 0 & - \\
\hline 钝吻棒花鱼 (Abbotina obtusirostris) & 13 & 80 & 6.2 & 13 & 0 & - \\
\hline 圆口铜鱼 (Coreius guichenoti) & 2 & 46.5 & 23.3 & 2 & 0 & - \\
\hline 墨头鱼 ( Garra pingi) & 19 & 1229.2 & 64.7 & 19 & 0 & - \\
\hline 中华倒刺鲃( Spinibarbus sinensis) & 4 & 658.8 & 164.7 & 4 & 0 & - \\
\hline 齐口裂腹鱼 (Schizothorax prenanti) & 163 & 17299.7 & 106.1 & 163 & 24 & 480.0 \\
\hline 短须裂腹鱼 (Schizothorax wangchiachii) & 123 & 9841.6 & 80.0 & 123 & 17 & 339.4 \\
\hline 昆明裂腹鱼 (Schizothorax grahami) & 1 & 409.6 & 409.6 & 1 & 0 & - \\
\hline 鲫 ( Carassius auratus) & 5 & 243.8 & 48.8 & 5 & 1 & 56.7 \\
\hline 犁头鳅( Lepturichthys fimbriata) & 23 & 55.7 & 2.4 & 23 & 16 & 3.2 \\
\hline 中华金沙鲀( Jinshaia sinensis) & 4 & 43.5 & 10.9 & 4 & 0 & - \\
\hline 短身金沙鱾( Jinshaia abbreviata) & 170 & 680.6 & 4.0 & 170 & 11 & 8.1 \\
\hline 峨嵋后平鳅 (Metahomaloptera omeiensis) & 2 & 3.3 & 1.7 & 2 & 0 & - \\
\hline 鲇( Silurus asotus) & 12 & 809.3 & 67.4 & 12 & 0 & - \\
\hline 黄颡鱼 (Pelteobagrus fulvidraco) & 152 & 692.7 & 4.6 & 152 & 8 & 21.6 \\
\hline 瓦氏黄颡鱼( Pelteobagrus vachelli) & 2 & 105.7 & 52.9 & 2 & 0 & - \\
\hline 粗唇鮠( Leiocassis crassilabris) & 2 & 69.9 & 35.0 & 2 & 0 & - \\
\hline 凹尾拟魭(Pseudobagrus emarginatus) & 347 & 1853.7 & 5.3 & 290 & 108 & 11.4 \\
\hline 白缘鱼央 ( Liobagrus marginatus) & 54 & 690 & 12.8 & 42 & 12 & 17.0 \\
\hline 中华纹胸鮡( Glyptothorax sinense) & 78 & 1633.1 & 20.9 & 78 & 24 & 11.2 \\
\hline 黄石爬鮡( Euchiloglanis kishinouyei) & 10 & 211.7 & 21.2 & 10 & 0 & - \\
\hline 子陵吻鰕虎 (Rhenogobius giurinus) & 1 & 2.6 & 2.6 & 1 & 0 & - \\
\hline
\end{tabular}


聚类分析结果表明:在 $56.55 \%$ 的 Bray-Curtis 相 似性水平上可将监测区域 12 个月的鱼类优势种群 聚结构分为两组: 组 1 包括 $1-6$ 月和 $11-12$ 月的 群聚结构; 组 2 包括 7-10 月的群聚结构 (图 2). One-way ANOSIM 检验显示组 1 和组 2 的群聚结构 在统计学上差异显著 ( 全局 $R=0.982, P=0.002$, 迭 代次数 495 次时满足软件收玫要求).

\section{2 各组的指示种组成及其性成熟个体丰度在不同 组的差异}

共有 8 种鱼类可以作为黑水河下游 2014 年各 聚类组的指示种 (表 2), 其中组 1 有指示种 3 种, 分 别为齐口裂腹鱼、短体副鳅和红尾副鳅, 组 2 有指示 种 5 种, 分别为前鯺高原鳅、凹尾拟鲿、犁头鱾、短须 裂腹鱼和大鳞副泥鳅. 这些鱼类中, 齐口裂腹鱼、短 体副鳅、前鯺高原䱊和短须裂腹鱼均为长江上游特 有鱼类.

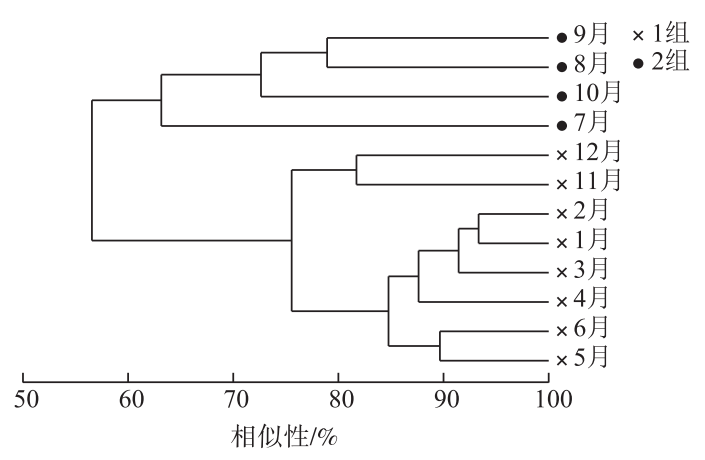

图 22014 年 $1-12$ 月黑水河下游 鱼类群聚结构的聚类分析

Fig. 2 Cluster analysis of fish assemblage structures in the lower reaches of the Heishui River from January to December of 2014

表 2 各聚类组的指示种及其指示值和各指示种在不同聚类组中的丰度

Tab.2 Indicator species and their indicative values in two groups (grouped by cluster analysis) and the abundance of each indicator species in different groups

\begin{tabular}{|c|c|c|c|c|c|}
\hline 种类 & 聚类组 & 指示值 & $P$ 值 & 在组 1 中的平均丰度/(尾/h) & 在组 2 中的平均丰度/ (尾/h) \\
\hline 齐口裂腹鱼 & 1 & 0.914 & 0.002 & 1.72 & 0.61 \\
\hline 短体副鱾 & 1 & 0.824 & 0.006 & 41.12 & 8.79 \\
\hline 红尾副鳅 & 1 & 0.728 & 0.004 & 25.89 & 9.69 \\
\hline 前鯺高原鳅 & 2 & 0.974 & 0.020 & 1.43 & 53.54 \\
\hline 凹尾拟魭 & 2 & 0.940 & 0.026 & 0.41 & 6.37 \\
\hline 犁头鳅 & 2 & 0.884 & 0.003 & 0.09 & 0.67 \\
\hline 短须裂腹鱼 & 2 & 0.736 & 0.017 & 0.66 & 1.85 \\
\hline 大鳞副泥鳅 & 2 & 0.612 & 0.015 & 0.09 & 0.14 \\
\hline
\end{tabular}

齐口裂腹鱼、短体副鳅、红尾副鳅和短须裂腹鱼 4 种指示种的性成熟个体的丰度分布范围 (极差) 和平 均值在组 1 大于组 2 , 其余 4 种鱼类( 前鯺高原鳅、凹尾拟鲿、犁头鳅和大鳞副泥鳅) 性成熟个体的分布范围 (极差) 和平均值均为组 1 小于组 2 (图 3 ).

\section{3 群聚结构变动与指示种性成熟个体丰度变动的关系}

DistlLM 的分析结果由两部分组成: 1) 边际检验: 显示单一预测变量与鱼类群聚结构变动的关系, 其结 果表明齐口裂腹鱼和犁头鲀性成熟个体丰度的逐月变动与鱼类群聚结构的逐月变动具有显著相关性 $(P<$ $0.05) ; 2$ ) 序列检验: 显示所有预测变量与鱼类群聚结构变动的关系, 其结果表明齐口裂腹鱼、大鳞副泥鳅和 犁头鳅的性成熟个体丰度的逐月变动与鱼类群聚结构的逐月变动具有显著相关性 $(P<0.05)($ 表 3$)$. 尽管如 此, 预测模型的选择结果表明: 除凹尾拟鲿以外, 其他 7 种鱼类加人模型时的 Adjusted $R^{2}$ 最大, 为 0.772 , 显示 这 7 种鱼类的性成熟个体丰度能够有效地预测黑水河下游鱼类群聚的丰度, 黑水河下游鱼类群聚的丰度会 随着这 7 种鱼类性成熟个体丰度的增加而增加.

dbRDA 分析结果如图 4 所示. 图中, 月份点垂直投影到轴 1 或轴 2 上,投影点位置为各月鱼类群聚结构 在轴 1 或轴 2 上的得分, 图中圆圈为半径为 1 的圆, 圆中直线 (向量) 长度代表某一预测变量与轴 1 和轴 2 的 Spearman 相关性. 总之, 图 4 显示: 1) 轴 1 能够解释鱼类群聚结构逐月变动总变异的 $65.1 \%$, 而轴 2 能够解释 总变异的 $14.5 \%$, 两轴共解释总变异的 $79.6 \%$; 2) 1-6月的鱼类群聚结构与轴 1 呈正相关, 但 7-12 月的鱼 类群聚结构与轴 1 呈负相关;3) 1 月和 3-7 月的鱼类群聚结构与轴 2 呈正相关, 但 2 月和 $8-12$ 月的鱼类 

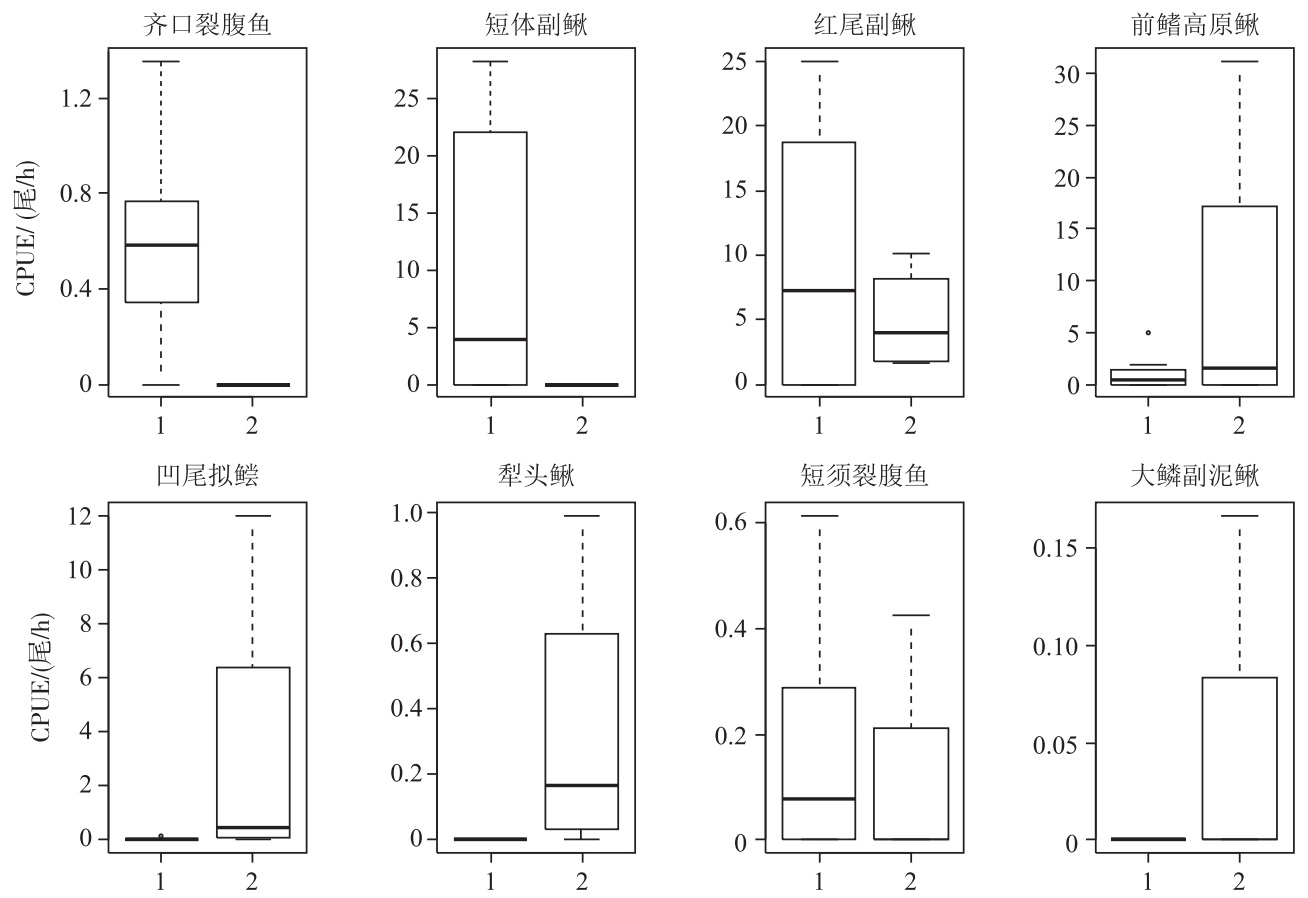

图 38 种指示种的性成熟个体在组 1 和组 2 中的丰度分布

Fig.3 Abundance distribution of sexually mature individuals for 8 indicator species in group 1 and group 2

表 3 基于 DistlLM 对群聚结构变动与指示种性成熟个体丰度变动的关系进行分析的结果

Tab.3 Results of relationships between monthly variations of abundance of sexual mature individuals for indicator species and monthly variations of fish assemblage structures by using DistlLM

\begin{tabular}{|c|c|c|c|c|c|c|}
\hline \multirow{2}{*}{$\begin{array}{l}\text { 预测变量 } \\
\text { (性成熟个体丰度) }\end{array}$} & \multicolumn{3}{|c|}{ 边际检验 } & \multicolumn{3}{|c|}{ 序列检验 } \\
\hline & 伪 $F$ 统计量 & $P$ & 解释变异比例 $/ \%$ & 伪 $F$ 统计量 & $P$ & 解释变异比例 $/ \%$ \\
\hline 齐口裂腹鱼 & 10.055 & $0.001^{*}$ & 52.51 & 10.055 & $0.001^{*}$ & 52.51 \\
\hline 大鳞副泥鳅 & 2.853 & 0.087 & 22.19 & 3.881 & $0.025^{*}$ & 14.31 \\
\hline 犁头鳅 & 4.420 & $0.003^{*}$ & 30.65 & 2.752 & $0.050^{*}$ & 8.49 \\
\hline 前鯺高原鳅 & 1.509 & 0.230 & 13.11 & 2.207 & 0.091 & 5.92 \\
\hline 红尾副鳅 & 0.760 & 0.484 & 7.06 & 1.349 & 0.270 & 3.45 \\
\hline 短须裂腹鱼 & 1.029 & 0.355 & 9.33 & 1.842 & 0.205 & 4.13 \\
\hline 短体副鳅 & 0.788 & 0.477 & 7.30 & 1.401 & 0.297 & 2.91 \\
\hline 凹尾拟魭 & 1.704 & 0.230 & 14.56 & & & \\
\hline
\end{tabular}

* 显著性水平 $P<0.05$.

群聚结构与轴 2 呈负相关;4) 1-6月鱼类群聚结构的丰度主要受齐口裂腹鱼和短体副鳅性成熟个体丰度增 加而短须裂腹鱼、大鳞副泥鳅、红尾副鲀、犁头鲀和前鯺高原鳅性成熟个体丰度减少的影响, 而 7- 12 月鱼 类群聚结构的丰度主要受短须裂腹鱼、大鳞副泥鳅、红尾副鲀、犁头鲀和前鯺高原鳅性成熟个体丰度增加而 齐口裂腹鱼和短体副鳅性成熟个体丰度减少的影响,即不同月份的鱼类丰度会随着 7 种鱼类产卵群体数量 的变动而变动; 5) 组 1 除受短体副鳅性成熟个体丰度正向影响的同时,也受前鯺高原鳅和短须裂腹鱼性成 熟个体丰度的负向影响,而组 2 的鱼类丰度受这 3 种鱼类性成熟个体丰度的影响与组 1 刚好相反. 


\section{3 讨论}

\section{1 产卵迁徙对黑水河下游鱼类群聚结构变动的影响}

研究表明,许多鱼类具有在产卵季节向干流或支流某些河段进行产卵迁徙的特征 ${ }^{[8-10,15]}$. 这种特征将导 致某些河段鱼类的季节性变动,进而在很大程度上影响局域鱼类群落的结构和功能 ${ }^{[10}{ }^{15,25]}$. 特别就较小支 流而言, 其鱼类群落结构的稳定以及季节性更替很可能严重依赖于干流鱼类向支流的产卵迁徙 ${ }^{[26]}$. 在本研 究中,伴随着鱼类群聚结构在雨季和旱季 ${ }^{[17]}$ 的显著分离 (图 2, 组 1 和组 2), 8 种指示种的性成熟个体丰度 也在黑水河下游雨季和早季间发生不同程度的变动 (图 3). DistlLM 结果进一步显示: 7 种指示种鱼类的性 成熟个体丰度的变动能够解释鱼类群聚结构变 动 $77.20 \%$ 的变异, 其中齐口裂腹鱼、大鳞副泥 鱾和犁头鱾性成熟个体丰度的变动是影响鱼类 群聚结构变动的 3 个最显著的因素 (表 3 ), 表 明黑水河下游鱼类, 特别是齐口裂腹鱼、大鳞副 泥鱾和犁头鱾的季节性产卵活动对该区域鱼类 的群聚结构造成了明显的影响. dbRDA 结果与 DistlLM 结果一致 (图 4).

已有研究表明,大坝 (即使坝高小于 $7.6 \mathrm{~m}$ 的小型大坝) 会对鱼类的群聚结构造成明显影 响, 不仅导致坝上区域鱼类的物种数、资源丰度 和 Shannon's 指数显著低于坝下江段, 而且会导 致坝上江段的鱼类群聚向以适应静缓流生境为 主的鱼类群聚变化 ${ }^{[27-28]}$, 从而使得坝上区域的 鱼类, 特别是喜流水性的鱼类, 难以或仅有少量 个体补充到坝下江段. 在本研究中, 调查区域上 游分布有多个首尾连接的梯级小水电 (坝高在 $5.0 \sim 18.0 \mathrm{~m}$ 之间 $)^{[17]}$, 且所有指示种均为适应 流水生境的种类 ${ }^{[29]}$, 因此在该区域的 8 种指示 种个体 (表 2) 应该难以在调查区域上游的梯级 水库群内形成大规模的种群, 并有效地下行过

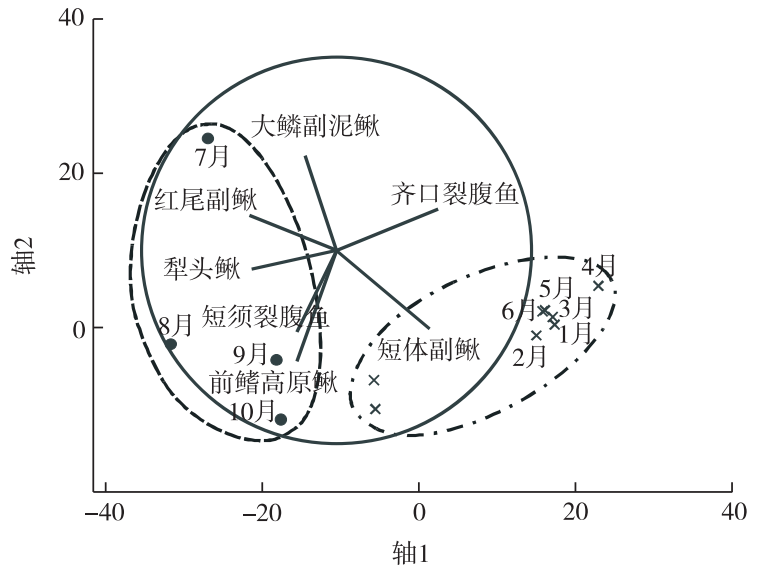

图 4 基于距离的圥余分析方法解析黑水河 下游鱼类群聚结构变动与指示种性成熟 个体丰度变动关系的可视化结果

Fig.4 Visualizing results of the relationships between the monthly variations of fish assemblage structure in the lower reaches of the Heishui River and the monthly variations of abundance of sexual mature individuals of indicator species by using the distancebased redundancy analysis (dbRDA) 坝达到本研究调查区域,因此这 8 种鱼类指示 种在调查区域的种群应该主要为黑水河下游 (老木河水电站以下) 和金沙江下游干流江段的种群. 各个指示 种在不同聚类组之间均显现明显丰度差异的特征 (表 2), 表明任一指示种在某一聚类组中的平均丰度均会 显著高于其在另一聚类组中的平均丰度. 显然, 这些鱼类在某一聚类组中丰度的明显增加 (表 2), 表明许多 个体在该聚类组对应的月份从金沙江干流迁徙到黑水河下游调查区域. 同时,结合性成熟个体丰度在不同 聚类组的变动 (图 3) 可知: 除短须裂腹鱼外, 齐口裂腹鱼、短体副䱊、红尾副鲉、前鯺高原䱊、凹尾拟魭、犁头 鲉和大鳞副泥鱾在某一聚类组中丰度增加的同时, 其性成熟个体的丰度也在该聚类组 (为这些鱼类的繁殖 季节) 呈增加的趋势. 以齐口裂腹鱼为例, 其为组 1 的指示种, 组 1 的丰度平均值大于组 2 ; 相应地, 组 1 的性 成熟个体丰度也大于组 2 , 而组 1 对应的月份 $\left(11-12\right.$ 月以及 $1-6$ 月) 为齐口裂腹鱼的繁殖季节 ${ }^{[30]}$. 上述这 种对应关系表明: 除短须裂腹鱼以外,其他 7 种指示种在黑水河下游的聚集是因为这些鱼类的产卵繁殖而 非仅因受食等因素导致的移动或迁徙. 许多研究也发现, 鱼类在繁殖时具有聚群的特征, 其性成熟个体在繁 殖季节在某一生境区域内的大量聚集表征了这些鱼类将在该区域进行产卵活动的极大可能性 ${ }^{[11,31-34]}$. 此 外, 就短须裂腹鱼而言, 其在组 1 中显示较低的总丰度 (表 2) 但显示较高的性成熟个体丰度 (图 3 ), 表明在 9 -10 月 (图 4, 圆中直线指向 9-10月) 就有较多的性成熟个体进人黑水河下游调查江段, 其产卵活动在黑水 河下游的发起时间很可能早于齐口裂腹鱼 (图 4); 同时, 短须裂腹鱼在组 2 中显示较高的总丰度 (表 2), 但 
显示较低的性成熟个体丰度 (图 3), 表明洄游到黑水河下游的许多短须裂腹鱼个体在组 2 包括的月份内, 有 许多个体很可能不能在该区域进行自然繁殖(产卵场面积有限)或许多为未成熟的个体 (表 1), 这仍需要进 行进一步研究.

\section{2 支流生境保护对金沙江下游鱼类种群维持的意义}

研究表明,鱼类群聚结构的动态变化除与鱼类的产卵迁徙有关以外,栖息地的环境条件也是影响其变 动的关键因素 ${ }^{[25,35-37]}$. 在局域尺度上, 表征物理生境特征的流速、底质组成、溶解氧、水深和河流宽度等参数 以及表征化学污染物的溶解氧浓度、电导率、总氮浓度、高锰酸盐指数等参数的显著变动通常能够对鱼类群 聚结构的变动产生明显影响 ${ }^{[25,36-37]}$. 此外, 就溪流鱼类而言, 溪流的空间位置 (溪流级别、下游量级等 ${ }^{[1,38]}$

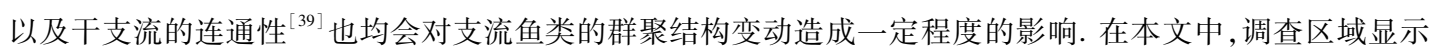
的受产卵迁徙明显影响的鱼类群聚变动模式 (表 2 和图 4), 表明调查区域具有较好的物理和化学生境特

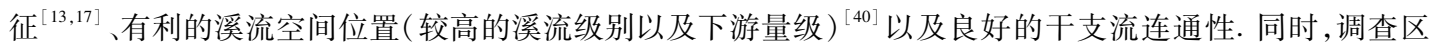
域分布有至少 12 种长江上游特有鱼类 (表 1), 是金沙江下游主要支流中除牛栏江以外特有鱼类分布最多的 支流 ${ }^{[13]}$. 因此, 维持黑水河下游现有的自然流水生境, 对该区域部分鱼类特别是长江上游特有鱼类生活史 的完成具有重要的意义. 许多研究也表明, 支流生境维持对部分鱼类生活史的完成具有重要的意义 ${ }^{[6,8,16,41]}$. 特别地,随着金沙江下游干流梯级水电站的陆续建设完工, 金沙江下游干流的绝大部分江段将成为静、缓流 的库区江段, 从而使得黑水河下游现有的自然生境变得更为重要. 张雄等报道,除黑水河外,金沙江下游还 分布有其他生境条件较好的支流,包括牛栏江、西溪河、普渡河、龙川江等 ${ }^{[13]}$, 这些河流的部分河段很可能与 黑水河下游河段一样,扮演着许多鱼类关键生境的角色. 甚至对于许多受水电开发而生境发生明显改变的 金沙江下游支流河段而言,其未开发前这些河段的生境也很可能是许多鱼类受食或繁殖的关键栖息地. 长 期以来, 由于缺乏对金沙江下游干支流关键鱼类生境 (特别是产粘性或沉性鱼卵鱼类的产卵场) 的详细调 查, 因此在比较不同江段的生境功能时, 常常无法确定支流对某些鱼类的重要生态学意义. 而且, 在对金沙 江下游水电开发对鱼类群聚结构影响的分析中, 通常着重考虑干流梯级开发对鱼类的影响, 而往往忽略支 流梯级开发对金沙江干流鱼类群聚结构的影响 ${ }^{[42-43]}$. 就本文而言, 7 种指示种鱼类在繁殖季节大量聚集到黑 水河下游产卵,表明这 7 种指示种鱼类能够在黑水河下游完成生活史周期, 并且其出生的后代有很大部分 能够补充到金沙江干流群体中, 因此黑水河下游生境的维持和保护对这些鱼类种群规模的维持具有重要的 意义. 而且, 研究表明,黑水河现有的小水电开发已对该区域的鱼类生境造成明显影响 ${ }^{[18]}$. 因此,在白鹤滩 蓄水前, 这 7 种指示种鱼类在黑水河的产卵生境面积也很可能已比小水电未修建前明显缩小. 由于这 7 种 鱼类是金沙江下游干流鱼类的重要组成部分, 因此黑水河产卵生境面积的缩小很可能对金沙江下游干流的 鱼类群聚结构造成明显影响. Wang 等也发现, 由于河流连通性的变化, 非淹没区的鱼类群聚结构也受到了 上下游大坝蓄水运行的影响 ${ }^{[44]}$.

张雄等报道,在金沙江下游一期水电工程完成以前,金沙江下游的 12 条支流已修建了大量水电站,而 且在未来,所有较大的 1 级支流将形成梯级水电群 ${ }^{[13]}$, 这对金沙江下游鱼类 (特别是仅局限分布在该区域的 鱼类) 的保护是非常不利的: 鱼类在干流和支流均无适宜面积的生境进行栖息繁衍. 就黑水河而言,尽管本 调查江段已作为白鹤滩库区干流鱼类的替代生境 (http: //www.cec.org.cn), 但是白鹤滩水电站蓄水到 $825 \mathrm{~m}$ 后,现有调查江段的大部分江段将变为永久淹没区或回水变动区; 该区域自然生境长度在白鹤滩蓄水运行 后的极其有限性, 决定了黑水河下游江段作为白鹤滩库区干流鱼类替代生境的有限作用. 已有研究表明, 只 有保持一定面积的关键栖息生境,鱼类才能够维持一定数量的种群规模而保持种群的延续 ${ }^{[45]}$, 因此为维持 现有的自然流水生境长度并发挥黑水河正常的替代生境功能, 黑水河小型水电站的拆除应是白鹤滩库区鱼 类保护优先选择的保护措施. 在此措施进行后, 采取其他措施对黑水河自然流水生境进行保护才可能发挥 其作为白鹤滩干流部分鱼类关键栖息地的作用.

\section{4 参考文献}

[ 1 ] Li YH, Yan YZ, Zhu R et al. Spatial variations in fish assemblages within the headwater streams of the Wanhe watershed: A river network-based approach. Journal of Fishery Sciences of China, 2014, 21(5) : 988-999. [李艳慧, 严云志, 朱仁 


\section{等. 基于河流网络体尺度的皖河河源溪流鱼类群落的空间格局. 中国水产科学, 2014, 21(5) : 988-999.]}

[ 2 ] Benda L, Poff NL, Miller D et al. The Network dynamics hypothesis: how channel networks structure riverine habitats. BioScience, 2004, 54(5): 413-427.

[ 3 ] Rodriguez-Iturbe I, Muneepeerakul R, Bertuzzo E et al. River networks as ecological corridors: a complex systems perspective for integrating hydrologic, geomorphologic, and ecologic dynamics. Water Resources Research, 2009, 45 (1): 1413-1434.

[ 4 ] Brown BL, Swan CM, Auerbach DA et al. Metacommunity theory as a multispecies, multiscale framework for studying the influence of river network structure on riverine communities and ecosystems. Freshwater Science, 2011, 30(1) : 310-327.

[ 5 ] Fukushima M. Damming in the Mekong River and its impacts on fish migration. Bmc Bioinformatics, 2011, 10(1): 1-11.

[ 6 ] Hladík M, Kubečka J. Fish migration between a temperate reservoir and its main tributary. Hydrobiologia, 2003, 504(1): 251-266.

[ 7 ] Walters DM, Zuellig RE, Crockett HJ et al. Barriers impedes upstream spawning migration of flathead chub. Transactions of the American Fisheries Society, 2014, 143(1): 17-25.

[ 8 ] Youngson AF, Jordan WC, Hay DW. Homing of Atlantic salmon (Salmo salar, L.) to a tributary spawning stream in a major river catchment. Aquaculture, 1994, 121(1/2/3) : 259-267.

[ 9 ] Nunn AD, Copp GH, Vilizzi L et al. Seasonal and diel patterns in the migrations of fishes between a river and a floodplain tributary. Ecology of Freshwater Fish, 2010, 19(1) : 153-162.

[10] Baxter CV. Fish movement and assemblage dynamics in a Pacific Northwest riverscape [Dissertation]. Corvallis: Oregon State University, 2002.

[11] Yin MC ed. Fish ecology. Beijing: China Agricultural Press, 1995: 105-269. [殷名称. 鱼类生态学. 北京: 中国农业出 版社, 1995: 105-269.]

[12] Armichardy DJ. The role of migration in community dynamics in Beaver Lake basin [Dissertation]. USA: Northern Michigan University, 2008: 55-82.

[13] Zhang X, Liu F, Lin PC et al. Habitat assessment and conservation priority for fishes in the lower Jinsha River. Resources and Environment in the Yangtze Basin, 2014, 23(4) : 496-503. [张雄, 刘飞, 林鹏程等. 金沙江下游鱼类栖息地评估 和保护优先级研究. 长江流域资源与环境, 2014, 23(4): 496-503.]

[14] Gosset C, Rives J, Labonne J. Effect of habitat fragmentation on spawning migration of brown trout (Salmo trutta L.).Ecology of Freshwater Fish, 2006, 15(3) : 247-254.

[15] Silva PS, Makrakis MC, Miranda LE et al. Importance of reservoir tributaries to spawning of migratory fish in the upper Paraná River. River Research \& Applications, 2015, 31(3) : 313-322.

[16] Meyer JL, Strayer DL, Wallace JB et al. The contribution of headwater streams to biodiversity in river networks. Jawra Journal of the American Water Resources Association, 2007, 43(1) : 86-103.

[17] Sun JN. Fish habitat simulation of the Heishui River-the backwater tributary of Baihetan reservoir [ Dissertation]. Hangzhou: Zhejiang University, 2013. [孙嘉宁. 白鹤滩水库回水支流黑水河的鱼类生境模拟研究 [学位论文]. 杭州: 浙 江大学, 2013.]

[18] Fu JJ, Huang B, Rui JL et al. Application of habitat simulation to fishery habitat protection in Heishui River. Journal of Hydroecology , 2016, 37(3) : 70-75. [傅菁菁, 黄滨, 芮建良等. 生境模拟法在黑水河鱼类栖息地保护中的应用. 水 生态学杂志, 2016, 37(3) : 70-75. ]

[19] Wang JW. Reproduction biology of Gobiocypris rarus. Acta Hydrobiologica Sinica, 1992, 16(2) : 165-174. [王剑伟. 稀有 鮈鲫的繁殖生物学. 水生生物学报, 1992, 16(2): 165-174.]

[20] Majewski AR, Lynn BR, Lowdon MK et al. Community composition of demersal marine fishes on the Canadian Beaufort Shelf and at Herschel Island, Yukon Territory. Journal of Marine Systems, 2013, 127(11) : 55-64.

[21] Siddig AAH, Ellison AM, Ochs A et al. How do ecologists select and use indicator species to monitor ecological change? Insights from 14 years of publication in Ecological Indicators. Ecological Indicators, 2016, 60 : 223-230.

[22] Dufrêne M, Legendre P. Species assemblages and indicator species definition: the need of an asymmetrical and flexible approach. Ecol Monogr, 1997, 67(3) : 345-366.

[23] Urban NA, Swihart RK, Malloy MC et al. Improving selection of indicator species when detection is imperfect. Ecological Indicators, 2012, 15(1): 188-197.

[24] Anderson MJ, Gorley RN, Clarke KR. PERMANOVA+for PRIMER: guide to software and statistical methods. Plymouth, 
UK, 2008.

[25] Li QF, Yan YZ, Chu L et al. Spatial and temporal patterns of stream fish assemblages within Taihu Basin. J Lake Sci, 2016, 8(6) : 1371-1380. DOI: 10.18307/2016.0623. [李其芳, 严云志, 储玲等. 太湖流域河流鱼类群落的时空分 布. 湖泊科学, 2016, 8(6): 1371-1380.]

[26] Taylor CM, Winston MR, Matthews WJ. Temporal variation in tributary and mainstem fish assemblages in a Great Plains stream system. Copeia, 1996, 1996(2) : 280-289.

[27] Fencl JS. How big of an effect do small dams have?: using ecology and geomorphology to quantify impacts of low-head dams on fish biodiversity [Dissertation]. Manhattan: Kansas State University, 2015.

[28] Holcomb JM, Nichols RB, Gangloff MM. Effects of small dam condition and drainage on stream fish community structure. Ecology of Freshwater Fish, 2016, 25(4) : 553-564.

[29] Yang SR. Fish community ecology in the Yangtze River Basin [Dissertation]. Beijing: University of Chinese Academy of Sciences, 2012. [杨少荣. 长江流域鱼类群落生态学研究 [学位论文]. 北京: 中国科学院大学, 2012.]

[30] Cheng F, Li W, Castello L et al. Potential effects of dam cascade on fish: lessons from the Yangtze River. Reviews in Fish Biology \& Fisheries, 2015, 25(3) : 569-585.

[31] Heyman WD, Kjerfve B, Graham RT et al. Spawning aggregations of lutjanus cyanopterus (cuvier) on the Belize Barrier Reef over a 6 year period. Journal of Fish Biology, 2005, 67(1) : 83-101.

[32] Domeier ML, Colin PL. Tropical reef fish spawning aggregations: defined and reviewed. Bulletin of Marine Science, 1997, 60(3) : 698-726.

[33] Karnauskas M, Chérubin LM, Paris CB. Adaptive significance of the formation of multi-species fish spawning aggregations near submerged capes. PloS One, 2011, 6(7) : e22067.

[34] Pears RJ, Choat JH, Mapstone BD et al. Reproductive biology of a large, aggregation-spawning serranid, Epinephelus fuscoguttatus (Forsskål) : management implications. Journal of Fish Biology, 2007, 71(3) : 795-817.

[35] Kapuscinski KL, Farrell JM. Habitat factors influencing fish assemblages at muskellunge nursery sites. Journal of Great Lakes Research, 2014, 40(1) : 135-147.

[36] Bonaca MO, Lipej L. Factors affecting habitat occupancy of fish assemblage in the Gulf of Trieste ( Northern Adriatic Sea). Marine Ecology, 2005, 26(1) : 42-53.

[37] Gao X, Zhang Y, Ding S et al. Response of fish communities to environmental changes in an agriculturally dominated watershed (Liao River Basin) in northeastern China. Ecological Engineering, 2015, 76: 130-141.

[38] Zhang XK, Wang HL, Wan A et al. Fishes in headwater streams of the Pihe River Basin: spatial distribution pattern and its main influencing factors. J Lake Sci, 2017, 29(1) : 176-185. DOI: 10.18307/2017.0119. [张晓可, 王慧丽, 万安 等. 㴓河流域河源溪流鱼类空间分布格局及主要影响因素. 湖泊科学, 2017, 29(1): 176-185.]

[39] Thornbrugh DJ. Influence of stream connectance and network spatial position on fish assemblage structure in the Kansas River basin, USA [Dissertation]. Manhattan: Kansas State University, 2008.

[40] Yan YZ, Zhan YJ, Chu L et al. Effects of stream size and spatial position on stream-dwelling fish assemblages. Acta Hydrobiologica Sinica, 2010, 34(5): 1022-1030. [严云志, 占姚军, 储玲等. 溪流大小及其空间位置对鱼类群落结构的 影响. 水生生物学报, 2010, 34(5): 1022-1030.]

[41] Kanno Y, Letcher BH, Coombs JA et al. Linking movement and reproductive history of brook trout to assess habitat connectivity in a heterogeneous stream network. Freshwater Biology, 2013, 59(1) : 142-154.

[42] Tang HY, Yang Z, Gao SB et al. Annual dynamics of fish biodiversity and community structure in the non-impounded reaches of the lower Jinsha River: case study of the Qiaojia section. Journal of Hydroecology, 2014, 35(6) : 7-15. [ 唐会元, 杨志, 高少波等. 金沙江下游巧家江段鱼类生物多样性及群落结构的年际动态. 水生态学杂志, 2014, 35(6): 7-15.]

[43] Fan XG, Wei QW, Chang JB et al. A review on conservation issues in the upper Yangtze River-a last chance for a big challenge: Can Chinese paddlefish (Psephurus gladius), Dabry's sturgeon, (Acipenser dabryanus) and other fish species still be saved? Journal of Applied Ichthyology, 2006, 22(s1) : 32-39.

[44] Wang L, Infante D, Lyons J et al. Effects of dams in river networks on fish assemblages in non-impoundment sections of rivers in Michigan and Wisconsin, USA. River Research \& Applications, 2011, 27(4) : 473-487.

[45] Benaka LR. Fish habitat: essential fish habitat and rehabilitation. The Quarterly Review of Biology, 2001, 76 (1): 1288-1289. 